\title{
$\lambda$-LEMMA FOR FAMILIES OF RIEMANN SURFACES AND THE CRITICAL LOCI OF COMPLEX HÉNON MAPS
}

\author{
TANYA FIRSOVA AND MIKHAIL LYUBICH
}

\begin{abstract}
We prove a version of the classical $\lambda$-lemma for holomorphic families of Riemann surfaces. We then use it to show that critical loci for complex Hénon maps that are small perturbations of quadratic polynomials with Cantor Julia sets are all quasiconformally equivalent.
\end{abstract}

\section{INTRODUCTION}

A holomorphic motion in dimension one is a family of injections $h_{\lambda}: A \rightarrow \hat{\mathbb{C}}$ of some set $A \subset \hat{\mathbb{C}}$ holomorphically depending on a parameter $\lambda$ (ranging over some complex manifold $\Lambda$ ). It turned out to be one of the most useful tools in onedimensional complex dynamics. First it was used to prove that a generic rational endomorphism $f: \hat{\mathbb{C}} \rightarrow \hat{\mathbb{C}}$ is structurally stable (see [12, 15]), and then has found numerous further applications.

Usefulness of holomorphic motions largely comes from their nice extension and regularity properties usually referred to as the $\lambda$-lemma. The simplest version of the Extension $\lambda$-lemma asserts that the holomorphic motion of any subset $X \subset$ $\hat{\mathbb{C}}$ extends to a holomorphic motion of the closure $\bar{X}$ [12,15. A more advanced version says that it extends to the whole Riemann sphere over a smaller parameter domain [5, 17]. The strongest version [16] asserts that if $\Lambda$ is the disk $\Delta \subset \mathbb{C}$, then the extension is globally defined over the whole $\Delta$. Moreover, the maps $h_{\lambda}$ are automatically continuous [12,15] and, in fact, quasiconformal [15].

In dimension two, holomorphic motions $h_{\lambda}: A \rightarrow \mathbb{C}^{2}, A \subset \mathbb{C}^{2}$, do not have such nice properties: in general, they do not admit extension even to the closure $\bar{A}$, and the maps $h_{\lambda}$ are not automatically continuous (let alone, quasiconformal). Still, under some circumstances, holomorphic motions turn out to be useful in higher dimensions as well; see [2,7].

In this paper, we prove a version of the $\lambda$-lemma for a class of holomorphic motions in $\mathbb{C}^{2}$ that naturally arise in the study of complex Hénon maps. (See also [6] for related versions.) Namely, we consider a holomorphic family of Riemann surfaces $S_{\lambda} \subset \mathbb{C}^{2}$ that fit into a complex two-dimensional manifold such that the boundaries of $S_{\lambda}$ move holomorphically in $\mathbb{C}^{2}$. We show that under suitable conditions, the holomorphic motion of the boundary can be extended to a holomorphic motion of the surfaces. The proof is based upon Teichmüller theory.

We use the holomorphic motion of the boundary of $S_{\lambda}$ to construct a holomorphic family in the Universal Teichmüller Curve, equipped with a holomorphic motion.

Received by the editors August 13, 2014 and, in revised form, April 9, 2016.

2010 Mathematics Subject Classification. Primary 32G15, 32H50, 37F10, 37F30.

The research of the second author was supported in part by NSF. 
We show that the total manifold of the fibers in the Universal Curve is isomorphic to the total manifold of $S_{\lambda}$. This gives us a desired holomorphic motion of $S_{\lambda}$.

This work is motivated by study of the geometry of the critical locus $\mathcal{C}$ for the Hénon automorphisms

$$
f:(x, y) \mapsto\left(x^{2}+c-a y, x\right)
$$

of $\mathbb{C}^{2}$. This locus was introduced by Hubbard (see [4]) as the set of tangencies between two dynamically defined foliations outside the "big" Julia set. It was studied in 9, 13 in the case of small perturbations (i.e., with a small Jacobian $a$ ) of one-dimensional hyperbolic polynomials $P_{c}: x \mapsto x^{2}+c$. In the case when $c$ is outside the Mandelbrot set (and $a$ is small enough), the critical locus has a rich topology described in 9 . Our version of the $\lambda$-lemma implies that all these critical loci are quasiconformally equivalent.

\section{BACKGROUND}

2.1. Notation. We will use the following notation throughout the paper: $\Delta$ for the unit disk, $\Delta_{\delta}$ for the disk of radius $\delta$ in the parameter plane, $\mathbb{H}$ for the hyperbolic plane, $D$ for the unit disk, $S$ for the unit circle, $\hat{\mathbb{C}}$ for the Riemann sphere.

2.2. $\lambda$-lemma. Let $M$ be a complex manifold, and let $\Delta \subset \mathbb{C}$ be a unit disk.

Definition 2.1. Let $A \subset M$. A holomorphic motion of $A$ over $\Delta$ is a map $f$ : $\Delta \times A \rightarrow M$ such that:

(1) For any $a \in A$, the map $\lambda \mapsto f(\lambda, a)$ is holomorphic in $\Delta$.

(2) For any $\lambda \in \Delta$, the map $a \mapsto f(\lambda, a)=: f_{\lambda}(a)$ is an injection.

(3) The map $f_{0}$ is the identity on $A$.

Holomorphic motions in one-dimensional dynamical context first appeared in [12,15]. The following simple but important virtues of one-dimensional holomorphic motions are usually referred to as the $\lambda$-lemma.

Extension $\lambda$-lemma $([12,15)$. Let $M=\hat{\mathbb{C}}, A \subset \hat{\mathbb{C}}$. Any holomorphic motion $f: \Delta \times A \rightarrow \hat{\mathbb{C}}$ extends to a holomorphic motion $\Delta \times \bar{A} \rightarrow \hat{\mathbb{C}}$.

Definition 2.2. Let $\left(X, d_{X}\right),\left(Y, d_{Y}\right)$ be two metric spaces. A homeomorphism $f: X \rightarrow Y$ is said to be quasisymmetric if there exists an increasing continuous function $\eta:[0, \infty) \rightarrow[0, \infty)$, such that for any triple of distinct points $x, y$ and $z$ :

$$
\frac{d_{Y}(f(x), f(y))}{d_{Y}(f(x), f(z))} \leq \eta\left(\frac{d_{X}(x, y)}{d_{X}(x, z)}\right) .
$$

A quasisymmetric map between two open domains is quasiconformal.

Qc $\lambda$-lemma ([15]). Under the circumstances of the Extension $\lambda$-lemma, for any $\lambda \in \Delta$, the map $f_{\lambda}: \bar{A} \rightarrow f_{\lambda}(\bar{A})$ is quasisymmetric.

Later, Bers \& Royden [5] and Sullivan \& Thurston [17] proved that there exists a universal $\delta>0$ such that under the circumstances of the Extension $\lambda$-lemma, the restriction of $f$ to the parameter disk $\Delta_{\delta}$ can be extended to a holomorphic motion $\Delta_{\delta} \times \hat{\mathbb{C}} \rightarrow \hat{\mathbb{C}}$ ("BRST $\lambda$-lemma"). Though this version of the $\lambda$-lemma will be sufficient for our dynamical applications, let us also state the strongest version asserting that $\delta$ is actually equal to 1 . 
Słodkowski's $\lambda$-lemma ([16]). Let $A \subset \hat{\mathbb{C}}$. Any holomorphic motion $f: \Delta \times A \rightarrow$ $\hat{\mathbb{C}}$ extends to a holomorphic motion $\Delta \times \hat{\mathbb{C}} \rightarrow \hat{\mathbb{C}}$.

In what follows, we will use the same notation $f$ for the extended holomorphic motion.

2.3. Elements of Teichmüller theory. We assume that the reader is familiar with the basics of Teichmüller theory. To set up terminology and notation, we recall some basic definitions and statements and refer to 14 for details.

Given a base Riemann surface $S$, let $\mathcal{Q C}(S)$ stand for the set of all Riemann surfaces quasiconformally equivalent to $R$.

Definition 2.3. Let $X_{1}, X_{2} \in \mathcal{Q C}(S)$, and let $\phi_{i}: S \rightarrow X_{i}$ be quasiconformal mappings. The pairs $\left(X_{1}, \phi_{1}\right)$ and $\left(X_{2}, \phi_{2}\right)$ are called Teichmüller equivalent if there exists a conformal isomorphism $\alpha: X_{1} \rightarrow X_{2}$ such that $\phi_{2}$ is homotopic to $\alpha \circ \phi_{1}$ relative to the ideal boundary $I(S)$. The class of equivalent pairs is called a marked by $S$ Riemann surface 1

Definition 2.4. The Teichmüller space $\mathcal{T}(S)$ modeled on $S$ is the space of marked by $S$ Riemann surfaces.

The space $\mathcal{T}(S)$ can be endowed with a natural Teichmüller metric.

Any marked Riemann surface $(\tilde{S}, \psi) \in \mathcal{T}(S)$ defines an isometry

$$
\psi_{*}: \mathcal{T}(S) \rightarrow \mathcal{T}(\tilde{S}), \quad \psi_{*}:(X, \phi) \rightarrow\left(X, \phi \circ \psi^{-1}\right),
$$

called a change of the base point of the Teichmüller space.

Definition 2.5. A Beltrami form $\mu$ on $S$ is a measurable $(-1,1)$-differential form with $\|\mu\|_{\infty}<1$. Accordingly, an infinitesimal Beltrami form $\nu$ on $S$ is a measurable $(-1,1)$-differential form with $\|\nu\|_{\infty}<\infty$.

Locally, $\mu$ can be represented as $\mu(z) \frac{d \bar{z}}{d z}$, where $\mu(z)$ is a measurable function with $|\mu(z)|<1$ a.e. (Notice that the latter condition is independent of the choice of the local coordinate.)

Any Beltrami form $\mu$ determines a conformal structure on $S$, i.e., the class of metrics conformally equivalent to $d z+\mu(z) d \bar{z}$. (In what follows, Beltrami forms and the corresponding conformal structures will be freely identified.) The standard structure $\sigma$ corresponds to $\mu \equiv 0$.

Let $\mathcal{B}(S)$ be the space of bounded Beltrami forms on $S$. It is identified with the unit ball in the complex Banach space $L^{\infty}(S)$, from which it inherits a natural complex structure.

Any quasiconformal map $f: S \rightarrow X$ induces the pullback

$$
f^{*}: \mathcal{B}(X) \rightarrow \mathcal{B}(S) \text {. }
$$

Measurable Riemann Mapping Theorem. Let $\mu$ be a bounded Beltrami form on $S$ with $\|\mu\|_{\infty}=k<1$. Then there exists a Riemann surface $S_{\mu} \in \mathcal{Q C}(S)$ and a $K$-quasiconformal map $f_{\mu}: S \rightarrow S_{\mu}$ with $K=(1+k) /(1-k)$ such that $f_{\mu}^{*} \sigma=\mu$. Moreover, it is unique up to postcomposition with some conformal map $h: S_{\mu} \rightarrow S_{\mu}^{\prime}$.

\footnotetext{
${ }^{1}$ Somewhat informally, we will use notation $(X, \phi)$, or just $X$, for the equivalence class.
} 
Analytically, $f=f_{\mu}$ gives a solution to the Beltrami equation

$$
\frac{\partial f}{\partial \bar{z}}=\mu \frac{\partial f}{\partial z} .
$$

By the Measurable Riemann Mapping Theorem, there is a natural projection

$$
\Phi_{S}: \mathcal{B}(S) \rightarrow \mathcal{T}(S) .
$$

The pullback operator from equation (2) descends to $f^{*}: \mathcal{T}(X) \rightarrow \mathcal{T}(S)$. It is the inverse of the change of the base point $f_{*}$.

Theorem 2.6. There exists a unique complex structure on $\mathcal{T}(S)$ such that the projection $\Phi_{S}$ is holomorphic.

Notice that the change of the base point (1) is a biholomorphism $\mathcal{T}(S) \rightarrow \mathcal{T}(\tilde{S})$, so the complex structure on the Teichmüller space is independent of the choice of $S$.

Let $U \subset S$ be a simply connected domain. Let $\mu$ be a Beltrami form in $U$. It is called harmonic if $\mu=\frac{\bar{q}}{\rho^{2}}$, where $q$ is a holomorphic quadratic differential and $\rho=\rho(z)|d z|$ is the hyperbolic metric in $U$.

Theorem 2.7 (BR $\lambda$-lemma). Let $f: \Delta \times A \rightarrow \hat{\mathbb{C}}$ be a holomorphic motion. There exists a unique extension $f: \Delta_{1 / 3} \times \hat{\mathbb{C}} \rightarrow \hat{\mathbb{C}}$ such that on each connected component of $\hat{\mathbb{C}} \backslash \bar{A}$, the Beltrami form $\mu_{\lambda}=\frac{\bar{\partial} f_{\lambda}}{\partial f_{\lambda}}$ is harmonic.

Let $S$ be a hyperbolic Riemann surface, and let $\phi: \mathbb{D} \rightarrow S$ be its universal covering with the group of deck transformations $\Gamma$.

Lemma 2.8 ([14, Lemma 6.6.3]). Let $\nu$ be an infinitesimal Beltrami form on $S$. Then $\nu \in \operatorname{Ker} d \Phi_{S}$ if and only if $\phi^{*} \nu=\bar{\partial} \eta$, where $\eta$ is a continuous $\Gamma$-invariant vector field on $\overline{\mathbb{D}}$ such that the distributional derivative $\bar{\partial} \eta$ has bounded $L^{\infty}$-norm and $\eta=0$ on $\mathbb{S}^{1}$.

Corollary 2.9. Assume that $S$ is a bounded type Riemann surface with the boundary $\partial S=\gamma^{1} \cup \cdots \cup \gamma^{n}$, where $\gamma^{i}$ are smooth Jordan curves. Let $\nu$ be an infinitesimal Beltrami form. Then $\nu \in \operatorname{Ker} d \Phi_{S}$ if and only if $\nu=\bar{\partial} \xi$, where $\xi$ is a continuous vector field on $S$ such that the distributional derivative $\bar{\partial} \xi$ has bounded $L^{\infty}$ norm and $\xi=0$ on $\partial S$.

Proof. Assume $\nu \in \operatorname{Ker} d \Phi_{S}$; then $\phi^{*} \nu=\bar{\partial} \eta$, where $\eta$ is a continuous $\Gamma$-invariant vector field on $\overline{\mathbb{D}}, \eta=0$ on $\mathbb{S}^{1}$, and $\bar{\partial} \eta$ has bounded $L^{\infty}$-norm. Its projection $\phi_{*} \eta$ is a continuous vector field on $S$, where $\phi_{*} \eta=0$ on $\partial S$ and $\bar{\partial}\left(\phi_{*} \eta\right)$ has bounded $L^{\infty}$-norm. To prove the other direction, assume that $\xi$ is a continuous vector field on $S$ so that the $L^{\infty}$-norm of $\bar{\partial} \xi$ is bounded and $\xi=0$ on $\partial S$. Let $\phi^{-1}(\xi)$ be a lift of the vector field $\xi$ to $\mathbb{D}$. Let $D$ be a fundamental domain of the group $\Gamma$. The vector field $\xi$ vanishes on the boundary. Therefore, $\left.\phi^{-1}(\xi)\right|_{D}$ is bounded in the hyperbolic metric. Since Möbius transformations preserve the hyperbolic metric, $\phi^{-1}(\xi)$ is bounded in the hyperbolic metric on $\mathbb{D}$. Thus, it vanishes on the boundary in the Euclidean metric.

The group $\Gamma$ is Fuchsian, so it acts on the whole Riemann sphere $\hat{\mathbb{C}}$. Let $\mathcal{B}^{\Gamma}(\hat{\mathbb{C}}) \subset$ $\mathcal{B}(\hat{\mathbb{C}})$ be the space of $\Gamma$-invariant Beltrami forms on $\hat{\mathbb{C}}$. We can map $\mathcal{B}(S)$ to $\mathcal{B}^{\Gamma}(\mathbb{C})$ by lifting $\mu \in \mathcal{B}(S)$ to the Beltrami form $\hat{\mu}=\phi^{*} \mu$ on $\mathbb{D}$ and then extending it by 
0 to the rest of $\hat{\mathbb{C}}$. By the Measurable Riemann Mapping Theorem, there exists a unique solution $\hat{f}_{\mu}: \hat{\mathbb{C}} \rightarrow \hat{\mathbb{C}}$ of the Beltrami equation (3) for $\hat{\mu}$, fixing 0,1 and $\infty$. It conjugates the Fuchsian group $\Gamma$ to a quasi-Fuchsian group $\Gamma_{\mu}$ preserving the quasidisk $\hat{f}_{\mu}(\mathbb{D})$.

Consider the map

$$
\Psi: \mathcal{B}(S) \times \hat{\mathbb{C}} \rightarrow \mathcal{B}(S) \times \hat{\mathbb{C}}, \quad(\mu, z) \mapsto\left(\mu, \hat{f}_{\mu}(z)\right) .
$$

The image $\Psi(\mathcal{B}(S) \times \mathbb{D})$ is an open subset of $\mathcal{B}(S) \times \hat{\mathbb{C}}$. Fiberwise actions of quasi-Fuchsian groups $\Gamma_{\mu}$ induce an action of $\Gamma$ on $\Psi(\mathcal{B}(S) \times \mathbb{D})$. For $\mu \in \mathcal{B}(S)$, the restriction of $\hat{f}_{\mu}$ to $\mathbb{S}^{1}$ and the group $\Gamma_{\mu}$ depend only on $\Phi_{S}(\mu) \in \mathcal{T}(S)$. We denote it by $\hat{f} \Phi_{S}(\mu)$. The quotient of $\Psi(\mathcal{B}(S) \times \mathbb{D})$ by the relation $\left[\left(\mu, \hat{f}_{\mu}\right) \sim\left(\nu, \hat{f}_{\nu}\right): \Phi_{S}(\mu)=\right.$ $\left.\Phi_{S}(\nu)\right]$ is a subset of $\mathcal{T}(S) \times \hat{\mathbb{C}}$, with a fiber-wise action of $\Gamma$. It is called the Bers fiber space. Factorizing the Bers fiber space by the action of $\Gamma$, we obtain the Universal Teichmüller Curve.

A holomorphic map $\gamma: \Delta \rightarrow \mathcal{T}(S)$ defines a map $\hat{f}_{\gamma(\lambda)}$ on $\mathbb{S}^{1}$. Let $h(\lambda, z):=$ $\hat{f}_{\gamma(\lambda, z)}$. The map $h$ is a holomorphic motion, $h: \Delta \times \mathbb{S}^{1} \rightarrow \hat{\mathbb{C}}$. We also use notation $h_{\lambda}(*):=h(\lambda, *)$.

Theorem 2.10 (Słodkowski's $\lambda$-lemma restated [8, [14, Proposition 6.10.5]). Every holomorphic map $\gamma: \Delta \rightarrow \mathcal{T}(S)$ lifts to a holomorphic map $\tilde{\gamma}: \Delta \rightarrow \mathcal{B}(S)$.

This implies that the holomorphic motion $h: \Delta \times \mathbb{S}^{1} \rightarrow \hat{\mathbb{C}}$ can be extended to a holomorphic motion $\tilde{h}: \Delta \times \overline{\mathbb{D}} \rightarrow \hat{\mathbb{C}}$, equivariant with respect to $\Gamma$.

\section{3. $\lambda$-LEMma For FAMiLIES of Riemann SURFACES}

Let us consider a complex 3-fold $\Delta \times \mathbb{C}^{2}$, and let $\pi_{1}: \Delta \times \mathbb{C}^{2} \rightarrow \Delta$ be the natural projection to $\Delta$. Let $\overline{\mathcal{S}} \subset \Delta \times \mathbb{C}^{2}$ be a complex 2-fold with non-empty boundary such that $\pi_{1}: \overline{\mathcal{S}} \rightarrow \Delta$ is a smooth locally trivial fibration with fibers $\bar{S}_{\lambda}$. We assume that the fibers $\bar{S}_{\lambda}$ are compact Riemann surfaces with boundary $\partial S_{\lambda}=\gamma_{\lambda}^{1} \cup \cdots \cup \gamma_{\lambda}^{n}$, where the $\gamma_{\lambda}^{i}$ are smooth Jordan curves that move holomorphically over $\Delta$. The intrinsic interior of $\overline{\mathcal{S}}$ is a complex 2 -fold $\mathcal{S}=\overline{\mathcal{S}} \backslash \partial \mathcal{S}$ that fibers over $\Delta$. The fibers are open Riemann surfaces

$$
S_{\lambda}=\operatorname{int} \bar{S}_{\lambda}=\bar{S}_{\lambda} \backslash \partial \bar{S}_{\lambda} .
$$

Note that since $\Delta$ is contractible, the fibration $\pi_{1}: \mathcal{S} \rightarrow \Delta$ is globally trivial in the smooth category.

Theorem 3.1. Let $f: \Delta \times \partial S_{0} \rightarrow \mathbb{C}^{2}$ be a holomorphic motion of $\partial S_{0}$ over $\Delta$ such that $\operatorname{Im} f_{\lambda}=\partial S_{\lambda}$. Moreover, assume that the maps $f_{\lambda}: \partial S_{0} \rightarrow \partial S_{\lambda}$ are diffeomorphisms. Then there exists a holomorphic motion $\tilde{f}$ of $\bar{S}_{0}$ over $\Delta$ such that:

(1) $f=\left.\tilde{f}\right|_{\partial S_{0}}$.

(2) For any $\lambda \in \Delta, \operatorname{Im} \tilde{f}_{\lambda}=S_{\lambda}$.

Note 3.2. Our argument, combined with the Bers-Royden $\lambda$-lemma [5], shows that over $\Delta_{1 / 3}$ there exists a canonical extension $\tilde{f}_{\lambda}$ as above (for which the Beltrami differentials $\mu_{\lambda}=\frac{\bar{\partial} f_{\lambda}}{\partial f_{\lambda}}$ are harmonic). 
We will show that the family $S_{\lambda}$ can be realized as a holomorphic curve in the Universal Curve over the Teichmüller space $\mathcal{T}\left(S_{0}\right)$.

Let us first extend the holomorphic motion $f$ to a smooth motion of $S_{0} \rightarrow S_{\lambda}$ over $\Delta$, for which we will use the same notation $f_{\lambda}$ as for the original motion. It defines a smooth curve $\tau_{\lambda}:=\left(S_{\lambda}, f_{\lambda}\right)$ in the Teichmüller space $\mathcal{T}\left(S_{0}\right)$.

Lemma 3.3. The elements $\tau_{\lambda} \in \mathcal{T}\left(S_{0}\right)$ do not depend on the choice of extension.

Proof. Let $f_{\lambda}$ and $g_{\lambda}$ be two extensions as above. Then

$$
g_{\lambda}^{-1} \circ f_{\lambda}: \bar{S}_{0} \rightarrow \bar{S}_{0},\left.\quad g_{\lambda}^{-1} \circ f_{\lambda}\right|_{\partial S_{0}}=\mathrm{Id}, \quad \lambda \in \Delta .
$$

Hence the maps $g_{\lambda}^{-1} \circ f_{\lambda}$ are homotopic to the identity rel $\partial S_{0}$, and thus define the same element of the Teichmüller space $\mathcal{T}\left(S_{0}\right)$.

Lemma 3.4. There exists a holomorphic 1-form $\omega$ on $S_{0}$ that extends smoothly to the boundary and $\omega(z) \neq 0$ for all $z \in \bar{S}_{0}$.

Proof. Let $\tilde{S}_{0}$ be the double of $S_{0}$ [1] There is a holomorphic embedding $\phi: S_{0} \rightarrow$ $\tilde{S}_{0}$ such that $\phi$ extends smoothly to the boundary $\partial S_{0}$. By the Riemann-Roch theorem, we can take a meromorphic form $u$ on $\tilde{S}_{0}$ such that zeros and poles of $u$ belong to $\tilde{S}_{0} \backslash \bar{S}_{0}$. The form $\omega=\left.u\right|_{S_{0}}$ is a desired holomorphic 1-form.

Theorem 3.5. The curve $\tau_{\lambda}$ is an analytic curve in $\mathcal{T}\left(S_{0}\right)$.

Proof. Let us show that $\frac{\partial \tau_{\lambda}}{\partial \bar{\lambda}}=0$. Fix some $\lambda_{0} \in \Delta$. Consider the map $f_{\lambda} \circ f_{\lambda_{0}}^{-1}$ : $S_{\lambda_{0}} \rightarrow S_{\lambda}$. This map defines a family $\mu_{\lambda}$ of Beltrami forms on $S_{\lambda_{0}}$ :

$$
\mu_{\lambda}=\frac{\bar{\partial}\left(f_{\lambda} \circ f_{\lambda_{0}}^{-1}\right)}{\partial\left(f_{\lambda} \circ f_{\lambda_{0}}^{-1}\right)} \in \mathcal{M}\left(S_{\lambda_{0}}\right) .
$$

Consider the projection map

$$
\Phi_{\lambda_{0}}: \mathcal{B}\left(S_{\lambda_{0}}\right) \rightarrow \mathcal{T}\left(S_{\lambda_{0}}\right)
$$

The map $\left(f_{\lambda_{0}}\right)^{*}$ provides an isomorphism between $\mathcal{T}\left(S_{\lambda_{0}}\right)$ and $\mathcal{T}\left(S_{0}\right)$. Moreover,

$$
\begin{gathered}
\left(f_{\lambda_{0}}\right)^{*} \circ \Phi_{\lambda_{0}}: \mathcal{M}\left(S_{\lambda_{0}}\right) \rightarrow \mathcal{T}\left(S_{0}\right), \\
\left(f_{\lambda_{0}}\right)^{*} \circ \Phi_{\lambda_{0}}\left(\mu_{\lambda}\right)=\tau_{\lambda} .
\end{gathered}
$$

Then we have

$$
\left.\frac{\partial \tau_{\lambda}}{\partial \bar{\lambda}}\right|_{\lambda=\lambda_{0}}=\left.d f_{\lambda_{0}}^{*} \circ d \Phi_{\lambda_{0}} \frac{\partial \mu_{\lambda}}{\partial \bar{\lambda}}\right|_{\lambda=\lambda_{0}} .
$$

Let us show that $\frac{\partial \mu_{\lambda}}{\partial \bar{\lambda}}\left(\lambda_{0}\right) \in \operatorname{Ker} d \Phi_{\lambda_{0}}$. To simplify the notation, we assume below that $\lambda_{0}=0$. We construct a vector field $\xi$ on $S_{0}$ such that $\frac{\partial \mu_{\lambda}}{\partial \bar{\lambda}}(0)=\bar{\partial} \xi$ and $\xi=0$ on $\partial S_{0}$ and apply Corollary [2.9, Let $\nu:=\frac{\partial \mu_{\lambda}}{\partial \lambda}(0), \kappa:=\frac{\partial \mu_{\lambda}}{\partial \bar{\lambda}}(0)$. Since $\mu_{0}=0$,

$$
\mu_{\lambda}=\lambda \nu+\bar{\lambda} \kappa+o(\lambda, \bar{\lambda})
$$

\footnotetext{
${ }^{2}$ The double of a Riemann surface is obtained by gluing the Riemann surface and its mirror image along the boundary.
} 
Let $\left(g_{1}, g_{2}\right): S_{0} \rightarrow \mathbb{C}^{2}$ be the defining functions of the Riemann surface $S_{0}$. The functions $g_{1}, g_{2}$ extend smoothly to the boundary, and

$$
f_{\lambda}=\left(\begin{array}{c}
g_{1}+\lambda u_{1}+\bar{\lambda} v_{1}+o(\lambda, \bar{\lambda}) \\
g_{2}+\lambda u_{2}+\bar{\lambda} v_{2}+o(\lambda, \bar{\lambda})
\end{array}\right)
$$

Since $f_{\lambda}$ is a holomorphic motion on the boundary, the functions $v_{1}, v_{2}$ are equal to zero on the boundary. Let $w$ be a local coordinate on $S_{0}, \partial f=\frac{\partial f}{\partial w} d w, \bar{\partial} f=$ $\frac{\partial f}{\partial \bar{w}} d \bar{w}$. By Lemma 3.4 there is a holomorphic non-zero 1-form $\omega$ on $S_{0}$ that extends smoothly to the boundary $\partial S_{0}$.

The functions $g_{1}$ and $g_{2}$ are holomorphic. Thus, $\partial g_{1}=h_{1} \omega, \partial g_{2}=h_{2} \omega$, where $h_{1}, h_{2}$ are holomorphic functions on $S_{0}$ that extend smoothly to $\partial S_{0}$.

$$
\begin{gathered}
\partial f_{\lambda}=\left(\begin{array}{c}
h_{1} \omega+\lambda \partial u_{1}+\bar{\lambda} \partial v_{1}+\ldots \\
h_{2} \omega+\lambda \partial u_{2}+\bar{\lambda} \partial v_{2}+\ldots
\end{array}\right), \quad \bar{\partial} f_{\lambda}=\left(\begin{array}{c}
\lambda \bar{\partial} u_{1}+\bar{\lambda} \bar{\partial} v_{1}+\ldots \\
\lambda \bar{\partial} u_{2}+\bar{\lambda} \bar{\partial} v_{2}+\ldots
\end{array}\right), \\
\mu_{\lambda} \partial f_{\lambda}=\bar{\partial} f_{\lambda}, \\
\left(\begin{array}{c}
\lambda+\bar{\lambda} \kappa+\ldots) \\
h_{1} \omega+\ldots \\
h_{2} \omega+\ldots
\end{array}\right)=\left(\begin{array}{c}
\lambda \bar{\partial} u_{1}+\bar{\lambda} \bar{\partial} v_{1}+\ldots \\
\lambda \bar{\partial} u_{2}+\bar{\lambda} \bar{\partial} v_{2}+\ldots
\end{array}\right) .
\end{gathered}
$$

Therefore,

$$
\kappa\left(\begin{array}{c}
h_{1} \omega \\
h_{2} \omega
\end{array}\right)=\left(\begin{array}{c}
\bar{\partial} v_{1} \\
\bar{\partial} v_{2}
\end{array}\right) .
$$

It follows from [18] that the space of maximal ideals in the algebra $A$ of the holomorphic functions on $S_{0}$ that extend continuously to the boundary is isomorphic to $\bar{S}_{0}$. The functions $h_{1}$ and $h_{2}$ do not have common zeros on $\bar{S}_{0}$. So the ideal generated by $h_{1}$ and $h_{2}$ coincides with $A$, in particular the function 1 belongs to the ideal. Hence there exists a pair of holomorphic functions $s_{1}$ and $s_{2}$ on $S_{0}$ that extend continuously to $\partial S_{0}$ so that $s_{1} h_{1}+s_{2} h_{2}=1$. Let $\eta$ be a holomorphic vector field on $S_{0}$ such that $\omega(\eta)=1$. Since $\omega$ extends smoothly to $\partial S_{0}, \eta$ extends smoothly to $\partial S_{0}$. Set

$$
\xi=\left(s_{1} v_{1}+s_{2} v_{2}\right) \eta
$$

then $\kappa=\bar{\partial} \xi$. Functions $v_{1}, v_{2}$ are smooth in $\bar{S}_{0}$, so $\bar{\partial} v_{1}, \bar{\partial} v_{2}$ are bounded in the $L^{\infty}$-norm. They are also equal to 0 on the boundary of $S_{0}$, so by Corollary 2.9 $\kappa \in \operatorname{Ker} d \Phi_{\lambda_{0}}$.

Let $\mathcal{R}$ be the total space of the lift of $\tau_{\lambda}$ to the Universal Teichmüller Curve.

Lemma 3.6. There exists a biholomorphism $\psi: \mathcal{R} \rightarrow \mathcal{S}$ that commutes with the projection: $\pi_{1} \circ \psi=\pi$.

Proof. Let $\mathbb{D}_{\lambda}$ be the fiber over $\tau_{\lambda}$ in the Bers fiber space. Let $h_{\lambda}$ be the holomorphic motion defined by $\tau_{\lambda}$ in the Bers fiber space. Let $\phi_{\lambda}: \mathbb{D}_{\lambda} \rightarrow S_{\lambda}$ be the uniformizing maps. By the construction we have the following equality on $\mathbb{S}^{1}$ :

$$
\phi_{\lambda} \circ h_{\lambda}=f_{\lambda} \circ \phi_{0} .
$$

We can consider it as a map $\phi_{\lambda}=\left(\phi_{\lambda}^{1}, \phi_{\lambda}^{2}\right): \mathbb{C} \rightarrow \mathbb{C}^{2}$.

By the Cauchy integral formula, we have

$$
\phi_{\lambda}^{j}(z)=\frac{1}{2 \pi i} \int_{\partial \mathbb{D}_{\lambda}} \frac{\phi_{\lambda}^{j}(\xi) d \xi}{\xi-z} .
$$


For a fixed $\lambda$, the map $f_{\lambda}$ is a diffeomorphism on each $\gamma_{i}$. The complement to the full preimage of $\gamma_{i}$ in $\mathbb{S}^{1}$ is the limit set of $\Gamma$. Hence it is a Cantor set of measure 0 . Therefore, the map $h_{\lambda}$ is absolutely continuous.

$$
\phi_{\lambda}^{j}(z)=\frac{1}{2 \pi i} \int_{\mathbb{S}^{1}} \frac{f_{\lambda}^{j}\left(\phi_{0}(\eta)\right) h_{\lambda}^{\prime}(\eta) d \eta}{h_{\lambda}(\eta)-z} .
$$

It follows that $\left(\phi^{1}, \phi^{2}\right)$ are holomorphic in $\lambda$. By definition, they are also holomorphic in $z$. Factorizing by the action of $\Gamma$, we get the required biholomorphism.

Proof of Theorem 3.1. The holomorphic motion $h_{\lambda}$ descends from the Universal Teichmüller Curve to the holomorphic motion of $S_{\lambda}$.

Applying the BR Extension $\lambda$-lemma, we get the canonical extension of the holomorphic motion over the disk of radius $1 / 3$.

Note 3.7. Let us note that Theorem 3.1 is still valid, with the same proof, if $\overline{\mathcal{S}}$ is embedded into $\Delta \times \mathbb{C}^{n}$ instead of $\Delta \times \mathbb{C}^{2}$. We can also give a more intrinsic version of it, without assuming that $\mathcal{S}$ is embedded into an ambient manifold, but assuming instead that the fibration extends beyond the boundary:

Theorem 3.8. Let $\mathcal{S}, \mathcal{R}$ be 2-dimensional complex manifolds, $\mathcal{S} \subset \mathcal{R}$. Let $\pi$ : $\mathcal{R} \rightarrow \Delta$ be a holomorphic map which is a locally trivial smooth fibration, and let $\pi^{-1}(\lambda)=R_{\lambda}$ be its fibers. Assume $\bar{S}_{\lambda}:=R_{\lambda} \cap \overline{\mathcal{S}}$ is a Riemann surface with boundary, consisting of finitely many smooth Jordan curves.

Let $f: \Delta \times \partial S_{0} \rightarrow \mathcal{R}$ be a holomorphic motion of $\partial S_{0}$ over $\Delta$. Moreover, assume that maps $f_{\lambda}: \partial S_{0} \rightarrow \partial S_{\lambda}$ are diffeomorphisms. Then there exists a holomorphic motion $\tilde{f}$ of $\bar{S}_{0}$ over $\Delta$ such that:

(1) $\left.\tilde{f}\right|_{\partial S_{0}}=f$.

(2) $\operatorname{Im}(\tilde{f})=\bar{S}_{\lambda}$.

Under these circumstances, we can still construct a curve in the universal Teichmüller space and the corresponding holomorphic motion in the same way as above. By Theorem [10, Th. 30.1], the normal bundle over $R_{\lambda_{0}}$ is trivial. Hence a neighborhood of $\bar{S}_{\lambda_{0}}$ in $\mathcal{R}$ can be embedded into $\mathbb{D} \times R_{\lambda_{0}}$, and therefore it can be embedded into $\mathbb{C}^{3}$. This is enough to carry through the above proofs of Theorem 3.5 and Lemma 3.6

\section{Application to dynamics}

4.1. Background on Hénon maps. Complex Hénon maps are biholomorphisms $f_{\lambda}: \mathbb{C}^{2} \rightarrow \mathbb{C}^{2}$ of the form

$$
f_{\lambda}\left(\begin{array}{l}
x \\
y
\end{array}\right)=\left(\begin{array}{c}
x^{2}+c-a y \\
x
\end{array}\right)
$$

where $\lambda=(a, c), a \in \mathbb{C}^{*}, c \in \mathbb{C}$.

In the one-dimensional holomorphic dynamics, the global phase portrait is to a large extent determined by the behavior of the critical points. Being diffeomorphisms, Hénon maps do not have critical points in the usual sense. However, they possess an interesting analogous object, the critical locus.

Let us recall the following dynamically significant sets:

$$
\begin{gathered}
U_{\lambda}^{+}=\left\{(x, y): f_{\lambda}^{n}(x, y) \rightarrow \infty \text { as } n \rightarrow+\infty\right\}, \quad K_{\lambda}^{+}=\mathbb{C}^{2} \backslash U_{\lambda}^{+}, \quad J_{\lambda}^{+}=\partial K_{\lambda}^{+}, \\
U_{\lambda}^{-}=\left\{(x, y): f_{\lambda}^{-n}(x, y) \rightarrow \infty \text { as } n \rightarrow+\infty\right\}, \quad K_{\lambda}^{-}=\mathbb{C}^{2} \backslash U_{\lambda}^{-}, \quad J_{\lambda}^{-}=\partial K_{\lambda}^{-},
\end{gathered}
$$




$$
J_{\lambda}=J_{\lambda}^{+} \cap J_{\lambda}^{-} .
$$

Domains $U_{\lambda}^{+}$and $U_{\lambda}^{-}$are called (forward and backward) escape loci; $J_{\lambda}$ is called the Julia set of the Hénon map.

In the one-dimensional polynomial dynamics, the critical points of the polynomial in the compliment of the filled Julia set are the critical point of Green's function. For a complex Hénon map, one can define the forward and backward Green's functions that measure the escape rate of the orbits under forward and backward iterations of the map [11:

$$
\begin{gathered}
G_{\lambda}^{+}(x, y)=\lim _{n \rightarrow \infty} \frac{\log ^{+}\left|f_{\lambda}^{n}(x, y)\right|}{2^{n}} \\
G_{\lambda}^{-}(x, y)=\lim _{n \rightarrow \infty} \frac{\log ^{+}\left|f_{\lambda}^{-n}(x, y)\right|}{2^{n}}+\log |a| .
\end{gathered}
$$

The functions $G_{\lambda}^{+}, G_{\lambda}^{-}$are pluriharmonic on the escape loci $U_{\lambda}^{+}, U_{\lambda}^{-}$respectively. Therefore, their level sets are foliated by Riemann surfaces. We denote by $\mathcal{F}_{\lambda}^{+}, \mathcal{F}_{\lambda}^{-}$ the corresponding foliations. These Riemann surfaces are in fact copies of $\mathbb{C}[11]$.

Let $p_{c}(x)=x^{2}+c$. As $a \rightarrow 0$, Hénon maps degenerate to a 1-dimensional map $x \mapsto p_{c}(x)$, acting on parabola $x=p_{c}(y)$, and $a \rightarrow 0$, Green's functions $G_{\lambda}^{+}$ converge to $G_{(0, c)}^{+}(x, y)=G_{p_{c}}(x)$, where $G_{p_{c}}(x)$ is the Green's function of the map $x \mapsto p_{c}(x)$.

There are also analogues $\phi_{\lambda,+}, \phi_{\lambda,-}$ of the Böttcher coordinates. The function $\phi_{\lambda,+}$ is well defined and holomorphic in a neighborhood $V_{\lambda}^{+}$of $(x=\infty, y=0)$ in the $\hat{\mathbb{C}}^{2}$-compactification of $\mathbb{C}^{2}$, and $\phi_{\lambda,+} \sim x$ as $x \rightarrow \infty$. Moreover, it semiconjugates $f$ to $z \mapsto z^{2}, \phi_{\lambda,+}\left(f_{\lambda}\right)=\phi_{\lambda,+}^{2}$.

In $V_{\lambda}^{+}$, the foliation $\mathcal{F}_{\lambda}^{+}$consists of the level sets of $\phi_{\lambda,+}$. It can be propagated to the rest of $U_{\lambda}^{+}$by the dynamics. One can also extend $\phi_{\lambda,+}$ to $U_{\lambda}^{+}$as a multi-valued function, and then use any branch of it to define $\mathcal{F}_{\lambda}^{+}$. Moreover, any branch is related to the Green's function by $G_{\lambda}^{+}=\log \left|\phi_{\lambda,+}\right|$.

The function $\phi_{\lambda,-}$ is defined in an analogous way.

\subsection{Critical locus.}

Definition 4.1. The critical locus $\mathcal{C}_{\lambda}$ is the set of tangencies between the foliations $\mathcal{F}_{\lambda}^{+}$and $\mathcal{F}_{\lambda}^{-}$.

The critical locus is given by the zeros of the 2 -form

$$
w=d \log \phi_{\lambda,+} \wedge d \log \phi_{\lambda,-} .
$$

It is a non-empty proper analytic subset of $U_{\lambda}^{+} \cap U_{\lambda}^{-}$which is invariant under the $\operatorname{maps} f_{\lambda}, f_{\lambda}^{-1}$.

Lyubich and Robertson ([13]) gave a description of the critical locus for Hénon mappings

$$
(x, y) \mapsto(p(x)-a y, x),
$$

where $p(x)$ is a hyperbolic polynomial with the connected Julia set and $a$ is sufficiently small. They showed that for each critical point $c$ of $p$ there is a component of the critical locus that is asymptotic to the line $y=c$. The rest of the components are iterates of these, and each is a punctured disk. In this case, all critical loci are obviously conformally equivalent. 
A topological description of the critical locus for complex Hénon maps that are perturbations of quadratic polynomials with disconnected Julia sets is given in [9]. The critical locus is a connected Riemann surface with rich topology. It is composed of countably many Riemann spheres $S_{n}$ with holes that are connected one to another by handles. There are $2^{k-1}$ handles between $S_{n}$ and $S_{n+k}$. On each sphere $S_{n}$ the handles accumulate to two Cantor sets.

We are ready to formulate the main result of this paper.

Theorem 4.2. The critical loci of the Hénon maps that are small perturbations of quadratic polynomials with disconnected Julia sets are quasiconformally equivalent.

4.3. Topological description of the critical locus. In this section we will give, following [9], a precise description of the critical locus.

Let $\mathcal{A}$ be the space of one-sided sequences of 0's and 1's ("infinite strings"), and let $\mathcal{A}^{n}$ be the space of $n$-strings of 0 's and 1's.

Let us describe truncated spheres that will serve as the building blocks for the critical locus. Consider a 2 -sphere $S \equiv S^{2}$ and a pair of disjoint Cantor sets $\Sigma, \Theta \subset S$. Let us fix a nest of figure-eight curves $\Gamma_{\alpha}^{n}$ and $L_{\alpha}^{n}, n=0,1,2, \ldots$, $\alpha \in \mathcal{A}^{n}$, respectively generating these Cantor sets in the following natural way 3

Let us start with a single figure-eight curve $\Gamma^{0}$ bounding two domains $D_{0}^{1}$ and $D_{1}^{1}$ (with an arbitrary assignment of labeling). The curve $\Gamma_{0}^{1} \subset D_{0}^{1}$ bounds two domains $D_{00}^{2}$ and $D_{01}^{2}$ compactly contained in $D_{0}^{1}$ (with an arbitrary assignment of the second label), and similarly, $\Gamma_{1}^{1} \subset D_{1}^{1}$ bounds two domains $D_{10}^{2}$ and $D_{11}^{2}$ inside $D_{1}^{1}$, etc. See Figure 1

We assume that $\bigcup_{\alpha} D_{\alpha}^{n} \supset \Sigma$ and $\operatorname{diam} D_{\alpha}^{n} \rightarrow 0$ as $n \rightarrow \infty$ (uniformly in $\alpha \in \mathcal{A}^{n}$ ), so for each sequence $\alpha \in \mathcal{A}$, there is a unique point

$$
\sigma_{\alpha}=\bigcap_{n=1}^{\infty} \overline{D_{\alpha_{n}}^{n}} \in \Sigma,
$$

where $\alpha_{n} \subset \mathcal{A}^{n}$ is the initial $n$-string of $\alpha$. This gives us a one-to-one coding of points $\sigma \in \Sigma$ by sequences $\alpha \in \mathcal{A}$.

Similarly, $\Theta$ is generated by a hierarchical nest of figure-eights $L_{\alpha}^{n}$. We assume that these two nests are disjoint in the sense that figure-eight $L^{0}$ lies in the unbounded component of $\mathbb{C} \backslash \Gamma^{0}$, and the other way around.

The singular points $\sigma_{\alpha}^{n}$ and $\theta_{\alpha}^{n}$ of the figure-eights $\Gamma_{\alpha}^{n}$ and $L_{\alpha}^{n}$ respectively are called their centers. For each figure-eight $\Gamma_{\alpha}^{n}$, select a disk $V_{\alpha}^{n} \ni \sigma_{\alpha}^{n}$ whose closure is disjoint from all other figure-eights $\Gamma_{\beta}^{m}$ and from $L^{0}$. Then select a disk $U_{\alpha}^{n} \ni \theta_{\alpha}^{n}$ with similar properties for each figure-eight $L_{\alpha}^{n}$. Moreover, make these choices so that the closures of all these disks are pairwise disjoint.

For each $n \in \mathbb{N}, \alpha \in \mathcal{A}^{n}$, we choose a homeomorphism $h_{\alpha}^{n}$ between the boundaries of $V_{\alpha}^{n}$ and $U_{\alpha}^{n}$. Finally, we mark a point $p \in S$ in the exterior of both figure-eights and the disks $\bar{U}^{0}, \bar{V}^{0}$. With all these choices in hand, we call

$$
S \backslash X \text {, where } X:=\Sigma \cup \Theta \cup\{p\} \bigcup_{n}\left(\bigcup_{\alpha \in \mathcal{A}^{n}} U_{\alpha}^{n} \cup V_{\alpha}^{n}\right) \text {, }
$$

a truncated sphere. Note that for any two truncated spheres $S \backslash X$ and $S^{\prime} \backslash X^{\prime}$ there is a homeomorphism $(S, X) \rightarrow\left(S^{\prime}, X^{\prime}\right)$ that restricts to the natural homeomorphisms between the corresponding marked sets.

\footnotetext{
${ }^{3}$ For $n=0$, we let $\mathcal{A}^{0}=\emptyset$.
} 


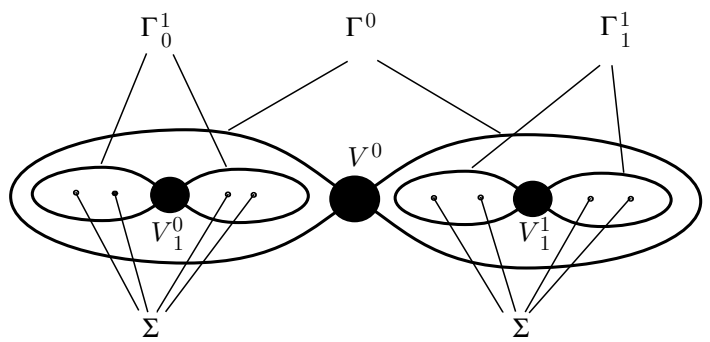

FiguRE 1. The geometry of a truncated sphere

Theorem 4.3. Assume that the quadratic polynomial $x \mapsto x^{2}+c$ has disconnected Julia set. Then there exists $\delta>0$ such that for any $|a|<\delta$ the critical locus of the Hénon map

$$
f_{\lambda}:\left(\begin{array}{c}
x \\
y
\end{array}\right) \mapsto\left(\begin{array}{c}
x^{2}+c-a y \\
x
\end{array}\right)
$$

is a non-singular Riemann surface that admits the following topological model. Take countably many copies $S_{m} \backslash X_{m}, m \in \mathbb{Z}$, of the truncated sphere $S \backslash X$, and glue the boundary of $V_{\alpha}^{n}$ of $S_{k}$ to the boundary of $U_{\alpha}^{n}$ of $S_{n+k+1}$ by means of the homeomorphism $h_{\alpha}^{n}$. The model map acts by translating $S_{n} \backslash X_{n}$ to $S_{n+1} \backslash X_{n+1}$.

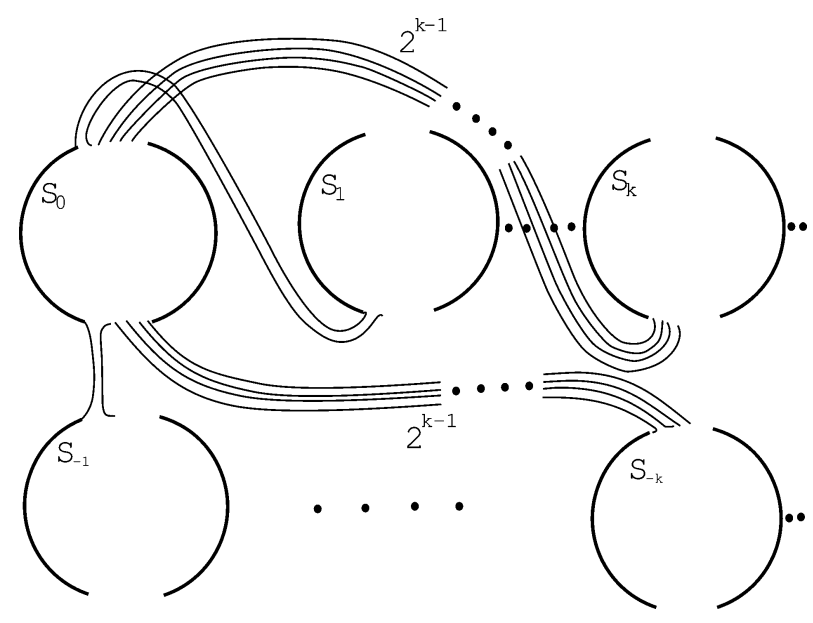

Figure 2. Critical locus

4.4. Proof of Theorem 4.2. The critical locus is a Riemann surface with infinitely generated fundamental group. Using results from [9], we subdivide the fundamental domain of the critical locus into pieces (each one is a disk with five holes) such that on the boundary of each piece we have a well-defined holomorphic motion. Then we extend the motion into each piece using Theorem 3.1. As a result, we get a holomorphic motion of all of the critical locus.

In [9] we gave a detailed description of the position of the critical locus $\mathcal{C}_{\lambda}$ in $\mathbb{C}^{2}$ for $\lambda \in \Lambda$, where $\Lambda$ is a set of parameters of a small perturbation of quadratic polynomials with disconnected Julia set. Below we fix a parameter $\lambda_{0}=\left(a_{0}, c_{0}\right) \in \Lambda$ 
and use the description from 9 to construct a holomorphic motion of the critical loci $\mathcal{C}_{\lambda}$, for $\lambda$ that belong to a 1-parameter family in a neighborhood of $\lambda_{0}$. Let us first describe a fundamental domain of the critical locus in $\mathbb{C}^{2}$. Let

$$
\begin{gathered}
\Omega_{\lambda}=\left\{(x, y) \in \mathbb{C}^{2}: \quad G_{a}^{+} \leq r, \quad|y| \leq \beta, \quad\left|p_{c}(y)-x\right|>|a| \beta\right\}, \\
\Upsilon_{\lambda}=\left\{(x, y) \in \mathbb{C}^{2}: G_{a}^{+}(x, y) \geq r, \quad|y| \leq \epsilon, \quad\left|p_{c}(y)-x\right|>|a| \beta\right\} .
\end{gathered}
$$

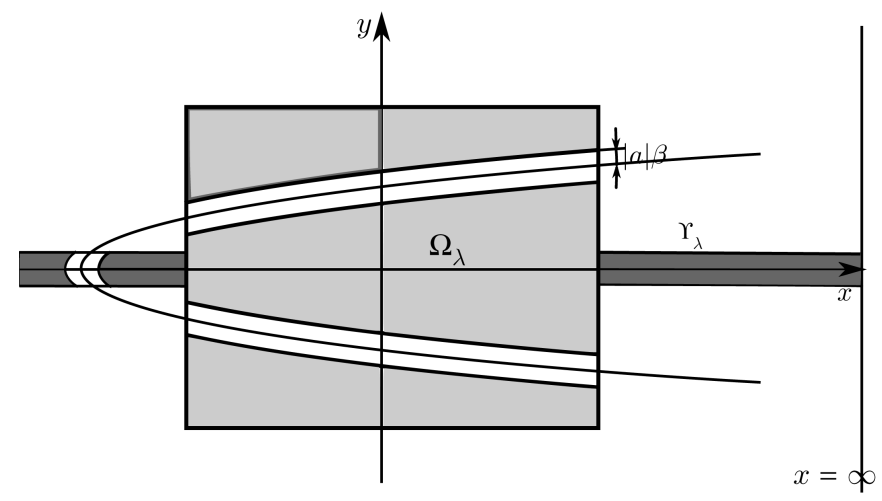

FiguRE 3. Domains $\Omega_{\lambda}$ and $\Upsilon_{\lambda}$

When $a \rightarrow 0$, domains $\Omega_{\lambda}$ converge in Hausdorff topology to $\Omega_{(0, c)}$. In $[9$ we choose $r, \beta$ and $\epsilon$, depending on $c$, so that for $c^{\prime}$ close to $c$ and $a$ small enough, $\mathcal{C}_{\lambda} \cap\left(\Omega_{\lambda} \cup \Upsilon_{\lambda}\right)$ form a fundamental domain for the map $f_{\lambda}$ on the critical locus (see [9, Lemma 13.6]). We further cut $\Omega_{\lambda} \cap U_{\lambda}^{+}$into subdomains $\Omega_{\lambda}^{\alpha}$, where $\alpha$ goes over all finite diadic strings.

We recursively encode the $n$-th preimages $\xi_{\alpha}$ of 0 under the map $z \mapsto z^{2}+c$ by diadic $n$-strings $\alpha$. We assume that 0 itself is parametrized by $\emptyset$. Let $\alpha^{0}, \alpha^{1} \in \mathcal{A}^{n+1}$ be the strings obtained by adding 0,1 correspondingly to $\alpha$ on the right. We encode preimages of $\xi_{\alpha}$ by $\alpha^{0}$ and $\alpha^{1}$. Since each connected component of

$$
\left\{\frac{r}{2^{n+1}} \leq G_{p_{c}} \leq \frac{r}{2^{n}}\right\}
$$

contains a unique $n$-preimage of the critical point, they are encoded by diadic $n$ strings $\alpha$ as well:

$$
\Omega_{(0, c)}^{\alpha}=\left\{\text { a connected component of }\left\{\frac{r}{2^{n+1}} \leq G_{(0, c)}^{+} \leq \frac{r}{2^{n}}\right\} \cap \Omega_{(0, c)}\right\}
$$

that contains a line $x=\xi_{\alpha}, \alpha \in \mathcal{A}^{n}$.

By the choice of $r$ in [9], the connected components of

$$
\left\{\frac{r}{2^{n+1}} \leq G_{\lambda}^{+} \leq \frac{r}{2^{n}}\right\} \cap \Omega_{\lambda}
$$

depend continuously on $a$ in the Hausdorff topology. We denote by $\Omega_{\lambda}^{\alpha}$ the continuation of $\Omega_{(0, c)}^{\alpha}$.

Let

$$
\begin{gathered}
\mathcal{C}_{\lambda}^{\alpha}:=\mathcal{C}_{\lambda} \cap \Omega_{\lambda}^{\alpha}, \quad \mathcal{C}_{\lambda}^{\Upsilon}:=\mathcal{C}_{\lambda} \cap \Upsilon_{\lambda}, \\
\mathcal{C}_{\lambda}^{f}:=\bigcup_{\alpha} \mathcal{C}_{\lambda}^{\alpha} \cup \mathcal{C}_{\lambda}^{\Upsilon} .
\end{gathered}
$$




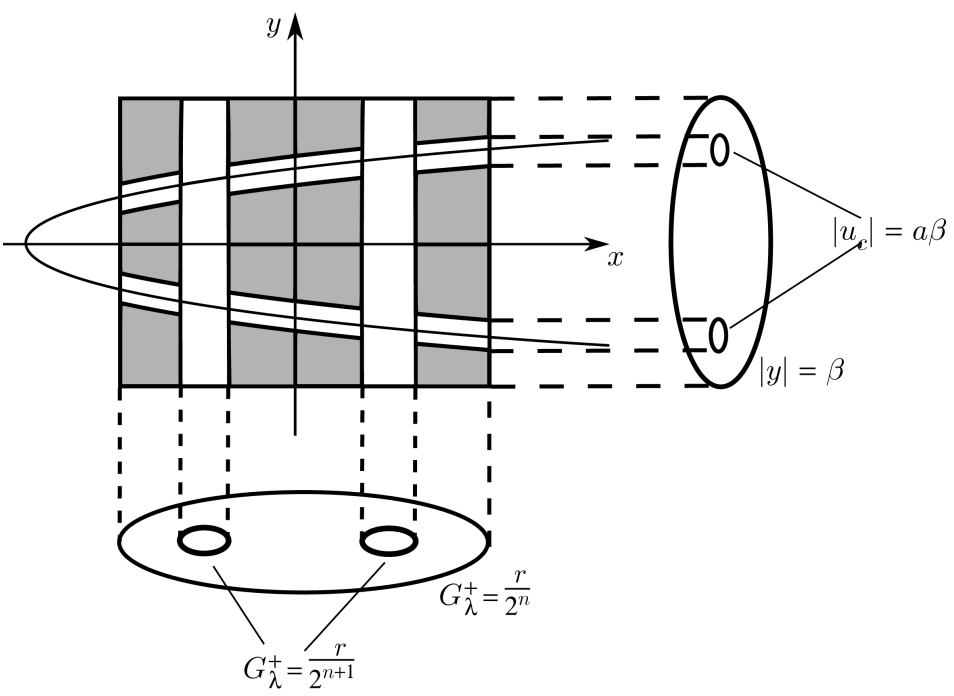

Figure 4. Domain $\Omega_{\lambda}^{\alpha}$

Since $\mathcal{C}_{\lambda}^{f}=\mathcal{C}_{\lambda} \cap\left(\Omega_{\lambda} \cup \Upsilon_{\lambda}\right), \mathcal{C}_{\lambda}^{f}$ is a fundamental domain of the critical locus under the map $f_{\lambda}$.

Let $u_{c}=y^{2}+c-x$.

Lemma 4.4 ([9, Lemma 11.4]). There exists $\delta$ such that for $\left|\lambda-\lambda_{0}\right|<\delta, \mathcal{C}_{\lambda}^{\alpha}$, where $\alpha \in \mathcal{A}^{n}, n=0,1, \ldots$, is a connected sum of two disks $D_{1}$ and $D_{2}$ with two holes each. The boundary of $D_{1}$ belongs to $\{|y|=\beta\}$, and the holes of $D_{1}$ have boundaries on $\left\{\left|u_{c}\right|=|a| \beta\right\}$. The boundary of $D_{2}$ belongs to $\left\{G_{\lambda}^{+}=\frac{r}{2^{n}}\right\}$ and the holes to $\left\{G_{\lambda}^{+}=\frac{r}{2^{n+1}}\right\}$.

Let

$$
\begin{gathered}
\gamma_{\lambda}^{\alpha}:=C_{\lambda}^{\alpha} \cap\{|y|=\beta\}, \\
\tilde{\gamma}_{\lambda}^{\alpha}:=f_{\lambda}\left(\gamma_{\lambda}^{\alpha}\right), \\
\delta_{\lambda}^{\alpha}:=C_{\lambda}^{\alpha} \cap\left\{G_{\lambda}^{+}=\frac{r}{2^{n}}\right\} .
\end{gathered}
$$

Then

$$
\partial \mathcal{C}_{\lambda}^{\alpha}=\gamma_{\lambda}^{\alpha} \cup \tilde{\gamma}_{\lambda}^{\alpha^{0}} \cup \tilde{\gamma}_{\lambda}^{\alpha^{1}} \cup \delta_{\lambda}^{\alpha} \cup \delta_{\lambda}^{\alpha^{0}} \cup \delta_{\lambda}^{\alpha^{1}}
$$

Lemma 4.5 ([9, Lemma 13.1]). There exists $\delta$ such that for $\left|\lambda-\lambda_{0}\right|\left\langle\delta, \mathcal{C}_{\lambda}^{\Upsilon}\right.$ is a punctured disk, with a hole removed. The puncture is at the point $(\infty, 0)$ and the boundary of the hole belongs to $\left\{\left|p_{c}(y)-x\right|=|a| \beta\right\}$.

$$
\partial \mathcal{C}_{\lambda}^{\Upsilon}=\tilde{\gamma}_{\lambda}^{\emptyset} \cup \delta_{\lambda}^{\emptyset} \cup(\infty, 0) .
$$

Let $D_{\delta}\left(\lambda_{0}\right)$ be a $\delta$-neighborhood of $\lambda_{0}$ in the parameter space.

Lemma 4.6. Let $D$ be a holomorphic disk in $D_{\delta}\left(\lambda_{0}\right)$. Then there exists a holomorphic motion of $\gamma_{\lambda}^{\alpha}$ and $\delta_{\lambda}^{\alpha}$ over $D$.

Proof. By [9, Lemma 11.1], in a neighborhood of $\delta_{\lambda}^{\alpha}, \mathcal{C}_{\lambda}$ is a graph of function $y_{\lambda}$ of $\left(\phi_{\lambda,+}^{2^{n}}\right)^{-1}$. Hence we can construct a holomorphic motion of $\delta_{\lambda}^{\alpha}$ by following 


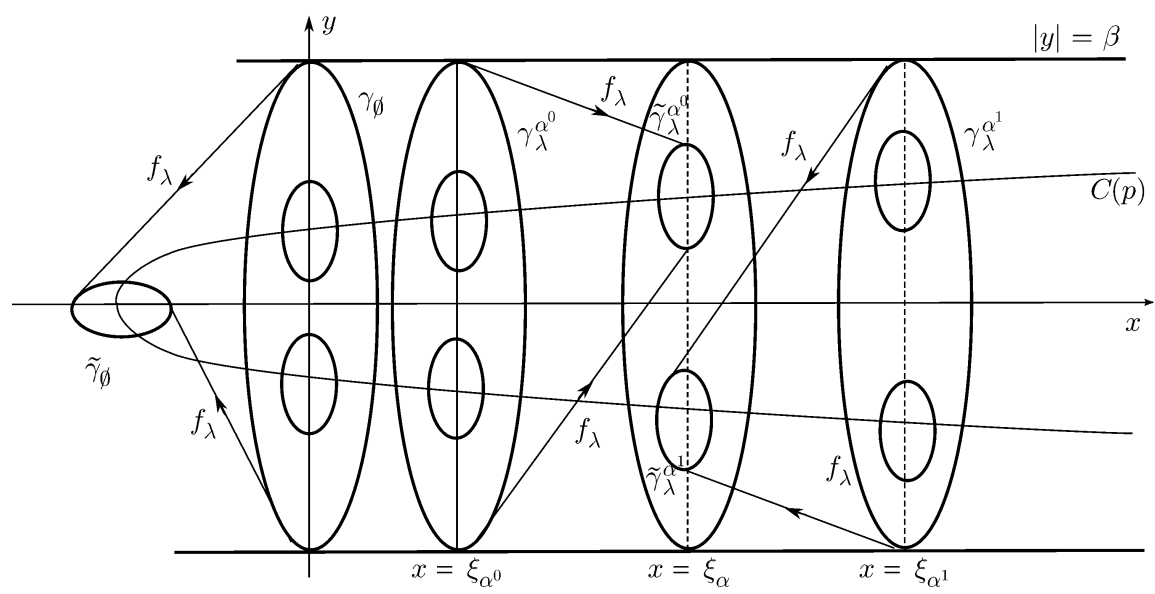

Figure 5. Pairing of boundary curves in the fundamental domain

the values of $\phi_{\lambda,+}^{2^{n}}$ : we map the point $\left(\left(\phi_{\lambda_{0},+}^{2^{n}}\right)^{-1}(z), y_{\lambda_{0}}\left(\left(\phi_{\lambda_{0},+}^{2^{n}}\right)^{-1}(z)\right)\right)$ on $C_{\lambda_{0}}$ (where $\left.|z|=e^{\frac{r}{2^{n}}}\right)$ to the point $\left(\left(\phi_{\lambda,+}^{2^{n}}\right)^{-1}(z), y_{\lambda}\left(\left(\phi_{\lambda,+}^{2^{n}}\right)^{-1}(z)\right)\right)$ on $C_{\lambda}$. Similarly, in a neighborhood of $\gamma_{\lambda}^{\alpha}, \mathcal{C}_{\lambda}$ is a graph of a function of $y$. Therefore, we can follow the values of $y$ to construct the holomorphic motion of $\gamma_{\lambda}^{\alpha}$.

Lemma 4.7. Let $D$ be a holomorphic disk in $D_{\delta}\left(\lambda_{0}\right)$. Then there exists a holomorphic motion of $C_{\lambda}^{f}$ over $D$ that is equivariant under $f_{\lambda}$ on the boundary.

Proof. We propagate the holomorphic motion constructed in the previous lemma to $\tilde{\gamma}_{\lambda}^{\alpha}$ by the dynamics. Thus we get a holomorphic motion of $\partial \mathcal{C}_{\lambda}^{\alpha}, \alpha \in \mathcal{A}_{n}$, $n=0,1, \ldots$, and $\partial \mathcal{C}_{\lambda}^{\Upsilon}$.

Then for each fixed $\mathcal{C}_{\lambda}^{\alpha}, \alpha \in \mathcal{A}_{n}$, we apply Theorem 3.1. We obtain a holomorphic motion of $\mathcal{C}_{\lambda}^{\alpha}$ over $D$ that extends the holomorphic motion of the boundary $\partial \mathcal{C}_{\lambda}^{\alpha}$. We can extend the holomorphic motion to a neighborhood of $\infty$ on $\mathcal{C}_{\lambda}^{\Upsilon}\left(\mathcal{C}_{\lambda}^{\Upsilon} \cap\left\{G_{\lambda}^{+}<R\right\}\right)$ by following the values of $\phi_{\lambda,+}$. Let $\tilde{\mathcal{C}}_{\lambda}^{\alpha}=\mathcal{C}_{\lambda}^{\alpha} \cap\left\{G_{\lambda}^{+}<R\right\}$. $\tilde{\mathcal{C}}_{\lambda}^{\alpha}$ is a disk with two holes, so we can apply Theorem 3.1 to obtain a holomorphic motion of $\tilde{\mathcal{C}}_{\lambda}^{\Upsilon}$. Hence, we constructed a holomorphic motion of the fundamental domain $\mathcal{C}_{\lambda}^{f}$. It is equivariant by construction.

We propagate the holomorphic motion to the rest of $\mathcal{C}_{\lambda}$ by dynamics. The space $\Lambda$ is path connected. Therefore, the critical loci $\mathcal{C}_{\lambda}$ for all maps that are small perturbations of quadratic polynomials with disconnected Julia set are quasiconformally equivalent.

\section{ACKNowledgements}

The authors thank Alex Brudnyi for useful discussions and the referee for raising interesting questions. 


\section{REFERENCES}

[1] William Abikoff, The real analytic theory of Teichmüller space, Lecture Notes in Mathematics, vol. 820, Springer, Berlin, 1980. MR590044

[2] Giovanni Bassanelli and François Berteloot, Lyapunov exponents, bifurcation currents and laminations in bifurcation loci, Math. Ann. 345 (2009), no. 1, 1-23, DOI 10.1007/s00208008-0325-1. MR 2520048

[3] Eric Bedford and John Smillie, Polynomial diffeomorphisms of $\mathbf{C}^{2}$. V. Critical points and Lyapunov exponents, J. Geom. Anal. 8 (1998), no. 3, 349-383, DOI 10.1007/BF02921791. MR 1707733

[4] Eric Bedford and John Smillie, Polynomial diffeomorphisms of $\mathbf{C}^{2}$. VI. Connectivity of J, Ann. of Math. (2) 148 (1998), no. 2, 695-735, DOI 10.2307/121006. MR1668567

[5] Lipman Bers and H. L. Royden, Holomorphic families of injections, Acta Math. 157 (1986), no. 3-4, 259-286, DOI 10.1007/BF02392595. MR857675

[6] E. M. Chirka, Holomorphic motions and the uniformization of holomorphic families of Riemann surfaces (Russian, with Russian summary), Uspekhi Mat. Nauk 67 (2012), no. 6(408), 125-202, DOI 10.1070/RM2012v067n06ABEH004819; English transl., Russian Math. Surveys 67 (2012), no. 6, 1091-1165. MR.3075079

[7] Romain Dujardin and Mikhail Lyubich, Stability and bifurcations for dissipative polynomial automorphisms of $\mathbb{C}^{2}$, Invent. Math. 200 (2015), no. 2, 439-511, DOI 10.1007/s00222-0140535-y. MR.3338008

[8] C. J. Earle, I. Kra, and S. L. Krushkal', Holomorphic motions and Teichmüller spaces, Trans. Amer. Math. Soc. 343 (1994), no. 2, 927-948, DOI 10.2307/2154750. MR1214783

[9] Tanya Firsova, The critical locus for complex Hénon maps, Indiana Univ. Math. J. 61 (2012), no. 4, 1603-1641, DOI 10.1512/iumj.2012.61.4658. MR3085620

[10] Otto Forster, Lectures on Riemann surfaces, Graduate Texts in Mathematics, vol. 81, Springer-Verlag, New York-Berlin, 1981. Translated from the German by Bruce Gilligan. MR648106

[11] John H. Hubbard and Ralph W. Oberste-Vorth, Hénon mappings in the complex domain. I. The global topology of dynamical space, Inst. Hautes Études Sci. Publ. Math. 79 (1994), 5-46. MR 1307296

[12] M. Yu. Lyubich, Some typical properties of the dynamics of rational mappings (Russian), Uspekhi Mat. Nauk 38 (1983), no. 5(233), 197-198. MR718838

[13] M. Lyubich and J. Robertson, The critical locus and rigidity of foliations of complex Hénon maps, Unpublished manuscript (2004).

[14] John Hamal Hubbard, Teichmüller theory and applications to geometry, topology, and dynamics. Vol. 1, Matrix Editions, Ithaca, NY, 2006. Teichmüller theory; With contributions by Adrien Douady, William Dunbar, Roland Roeder, Sylvain Bonnot, David Brown, Allen Hatcher, Chris Hruska and Sudeb Mitra; With forewords by William Thurston and Clifford Earle. MR2245223

[15] R. Mañé, P. Sad, and D. Sullivan, On the dynamics of rational maps, Ann. Sci. École Norm. Sup. (4) 16 (1983), no. 2, 193-217. MR732343

[16] Zbigniew Slodkowski, Holomorphic motions and polynomial hulls, Proc. Amer. Math. Soc. 111 (1991), no. 2, 347-355, DOI 10.2307/2048323. MR1037218

[17] Dennis P. Sullivan and William P. Thurston, Extending holomorphic motions, Acta Math. 157 (1986), no. 3-4, 243-257, DOI 10.1007/BF02392594. MR857674

[18] Michael Voichick, Ideals and invariant subspaces of analytic functions, Trans. Amer. Math. Soc. 111 (1964), 493-512. MR0160920

Department of Mathematics, Kansas State University, Manhattan, Kansas 66506

E-mail address: tanyaf@math.ksu.edu

Institute for Mathematical Sciences, Stony Brook University, Stony Brook, New YORK 11794

E-mail address: mlyubich@math.stonybrook.edu 